The Journal of Engineering and Exact Sciences - JCEC, Vol. 04 N. 04 (2018)

journal homepage: http://jcec.ufv.br

doi: https://doi.org/10.18540/jcecvl4iss4pp0405-0411

OPEN ACCESS - ISSN: 2527-1075

\title{
UTILIZAÇÃO DO BAMBU COMO MATÉRIA-PRIMA NA OBTENÇÃO DE POLPA CELULÓSICA VOLTADA À FABRICAÇÃO DE PAPEL DE EMBALAGEM SACKRAFT
}

\section{USE OF BAMBOO AS ALTERNATIVE FEEDSTOCK IN THE OBTAINING OF CELLULOSE PULP FOR THE MANUFACTURE OF SACKRAFT PACKING PAPER}

\section{F. P. BORGES ${ }^{1},{ }^{*}$, J. L. COLODETTE ${ }^{2}$ e F. J. B. GOMES ${ }^{3}$}

${ }^{1}$ Universidade Federal de Viçosa, Departamento de Química, Viçosa, Minas Gerais, Brasil

${ }^{2}$ Universidade Federal de Viçosa, Departamento de Engenharia Florestal, Viçosa, Minas Gerais, Brasil

${ }^{3}$ Universidade Federal Rural do Rio de Janeiro, Departamento de Produtos Florestais, Seropédica, Rio de Janeiro, Brasil

${ }^{*}$ Felipe Pedersoli Borges. Universidade Federal de Viçosa, Departamento de Química, Viçosa, Minas Gerais, Brasil, Phone: +55 31997453949

e-mail: pedersoli.felipe@gmail.com (F.P. Borges).

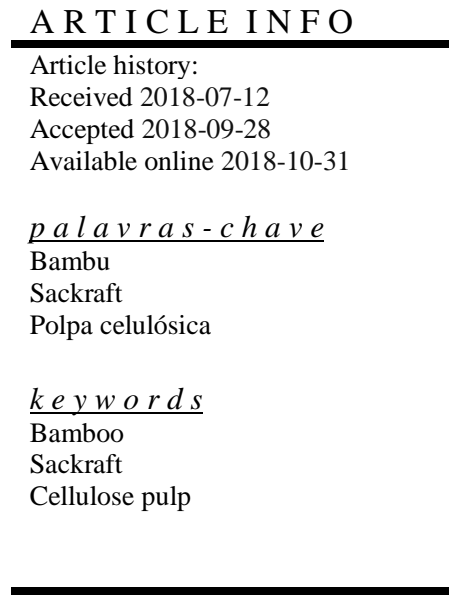

\begin{abstract}
A B S T R A C T
The increase in the cost of wood in recent years has stimulated the search for alternative feedstocks, and bamboo emerges as a potential biomass. Therefore, this study aims to analyze the applicability of bamboo of the species Bambusa vulgaris to the production of cellulose kraft pulp turned to the production of sackraft packing paper. The chemical and morphological characterization of the bamboo fibers and kraft pulping with starch-free bamboo were carried out. The bamboo presented levels of holocellulose (63.4\%) and lignin (23.2\%) favorable to its use in kraft pulping. The pulp yield $(54.9 \%)$ was higher than that obtained in conifers. The dimensions of bamboo fibers favor the obtaining of physicalmechanical characteristics required for the production of sackraft papers. It is, therefore, concluded that it is possible to use bamboo instead of wood in the suggested application.
\end{abstract}

\footnotetext{
R E S U M O

A tendência de aumento no custo da madeira nos últimos anos tem estimulado a busca por matérias-primas alternativas, onde o bambu surge como potencial biomassa. Sendo assim, esse estudo objetiva analisar a aplicabilidade do bambu da espécie Bambusa vulgaris para a produção de polpa celulósica kraft voltada à produção de papel de embalagem sackraft. Realizou-se a caracterização química e morfológica das fibras do bambu e polpação kraft com bambu livre de amido. O bambu apresentou teores de holocelulose $(63,4 \%)$ e lignina $(23,2 \%)$ favoráveis à sua utilização em polpação kraft. O rendimento de polpação $(54,9 \%)$ foi mais elevado que o obtido em coníferas. As dimensões das fibras do bambu favorecem as características físico-mecânicas necessárias para a produção de papéis do tipo sackraft. Conclui-se, portanto, ser possível utilizar o bambu em substituição à madeira na aplicação sugerida.
} 


\section{INTRODUÇÃO}

O eucalipto é a principal matéria-prima utilizada no setor de celulose e papel no Brasil atualmente, propiciando ao país a posição de segundo maior produtor de celulose do mundo, atrás apenas dos Estados Unidos (IBÁ, 2017). Quase 8 milhões de hectares são ocupados por plantios florestais no Brasil, dentre os quais 5,67 milhões são do gênero Eucalyptus sp. (IBA, 2017), correspondendo a $0,66 \%$ do território nacional. Cunha et al. (2018) destacam ainda que o clima favorável, a grande extensão territorial, a boa adaptabilidade do gênero aos solos brasileiros e as avançadas técnicas de silvicultura do eucalipto no Brasil são fatores que colaboram para a grande participação dessa matéria prima, sendo responsável por significativa fração do PIB industrial brasileiro. Entretanto, a produção de madeira tem apresentado tendências de aumento de custo no Brasil nos últimos anos, principalmente devido ao preço da terra. $\mathrm{O}$ relatório da Indústria Brasileira de Árvores, referente ao ano de 2015, demonstra que, nesse ano, a inflação indicativa a plantios florestais foi maior que a inflação nacional. O Índice Nacional de Custos de Atividade Florestal (INCAF-Poyry) foi de 12,8\%, $2,1 \%$ mais elevado que a inflação medida pelo Índice de Preços ao Consumidor (IPCA) para o mesmo período (IBÁ, 2017). Essa realidade pode ser observada durante todo o decorrer do século XXI até atualmente.

Nesse contexto, há uma busca constante por matériasprimas alternativas à madeira. Dentre as biomassas potenciais, o bambu surge como uma atrativa fonte, sendo considerada uma planta de origem tropical e subtropical recorrente em todos os continentes do planeta (XUHE, 2003). No Brasil, o uso do bambu como biomassa alternativa torna-se interessante pela grande quantidade de área plantada existente, sendo o país com maior área de bambu plantada na América (FILGUEIRAS e GONÇALVES, 2004; JUDZIEWICZ et al., 1999). Somente no estado do Acre estima-se que haja 7 milhões de hectares de bambus nativos, área semelhante à ocupada por plantios florestais em todo o Brasil (PEREIRA e BERALDO, 2007).

Beraldo e Azzini (2004) ressaltam que o rápido crescimento do bambu e as colheitas de ciclo curto, de 2 a 4 anos, além da característica perene dessa cultura, intensificam o interesse em sua aplicação. Além disso, o bambu apresenta boa capacidade de se desenvolver em solos pobres (REUBENS, 2010) e apresenta maior produtividade por hectare que os gêneros tradicionalmente utilizados na indústria de celulose e papel (OSTAPIV, 2007). De fato, os plantios de bambu crescem em torno de $3 \%$ por ano, de maneira oposta aos plantios florestais de forma geral, que apresentam constantes reduções anuais (ZEHUI, 2003). Destaca-se no Brasil a espécie Bambusa vulgaris, já aplicada industrialmente para a produção de papel cartão pela empresa Itapagé. De forma geral, já se utiliza o bambu para a produção de móveis e componentes da indústria moveleira, produção de brotos comestíveis, artesanato, como agente de recuperação de solos e encostas e na construção civil, exemplificando-se a construção de andaimes, realização de acabamentos e utilização em estruturas inteiras, à exceção de fundações (OLIVEIRA, 2013; ORTHEY, 2015; PEREIRA, 2012; PEREIRA e BERALDO, 2007).
É importante salientar que, nos últimos 10 anos, os destaques em crescimento na indústria de papel brasileiro foram os papeis de embalagem e sanitários (tissue). Em 2015, por exemplo, o Brasil produziu cerca de 10,3 milhões de toneladas de papel, sendo que $53 \%$ desse total são referentes a papeis de embalagens corrugadas, fazendo dessa classe de papel uma das mais importantes para o mercado papeleiro brasileiro, juntamente dos papeis de imprimir e escrever (SILVA et al., 2017). Para que se confira as propriedades físico-mecânicas necessárias para um papel de embalagem com alta qualidade, utiliza-se a madeira de Pinus ssp., que apresenta fibras longas. Papeis de embalagem kraftliner utilizam polpa kraft de pinus não branqueada com número kappa de 80 a 110, enquanto que os papeis de embalagem sackraft utilizam polpa kraft de pinus não branqueada com número kappa variando de 35 a 60 (BRÄNNVALL e GÖRAN, 2009).

O bambu surge como potencial matéria-prima para esses tipos de papéis, uma vez que também apresenta fibras consideradas como longas, de forma semelhante à madeira de pinus. Ilvessalo-Pfaffli (1995) nota, inclusive, que a polpa celulósica de bambu pode apresentar maior resistência ao rasgo que a polpa de coníferas. As fibras longas e estreitas presentes no bambu permitem maiores superfícies de contato para interações entre fibras, o que corrobora para melhor desempenho físico-mecânico do papel produzido em comparação às fibras de eucalipto. A extração do amido presente nas células parenquimáticas do bambu, além de agregar valor ao processo, permite a otimização da produção de polpa celulósica, uma vez que o amido eleva o consumo de reagentes na etapa de polpação e reduz a conversão dos cavacos em polpa celulósica (AZZINI et al., 1987).

Nesse contexto, o objetivo desse estudo é analisar a aplicabilidade do bambu da espécie Bambusa vulgaris para a produção de polpa celulósica kraft voltada à produção de papel de embalagem sackraft a partir de análises de caracterização morfológica e química das fibras de bambu e rendimento de polpação kraft em comparação com os resultados obtidos em literatura para madeira de Pinus.

\section{MATERIAL E MÉTODOS}

Esse estudo foi realizado no Laboratório de Celulose e Papel da Universidade Federal de Viçosa. Devido à disponibilidade, já em escala comercial de Bambusa vulgaris, essa espécie é a escolhida para o estudo. Utilizou-se amostra de B. vulgaris coletada junto ao Campus da Universidade Federal de Viçosa, em Viçosa, Minas Gerais. As amostras de bambu foram transformadas em cavacos manualmente, classificados segundo norma apresentada na seção 2.2 deste manuscrito. Após serem secos à temperatura ambiente, os cavacos foram acondicionados em sacos de polietileno para a condução dos estudos propostos. A fração aceita para a produção de papel foi analisada segundo sua morfologia e caracterização química, sofrendo posterior extração de amido para aumento do rendimento de polpação, com o material fibroso livre de amido sendo direcionado à polpação kraft. 


\subsection{Análises morfológicas das fibras do bambu}

Análises anatômicas foram determinadas a partir da maceração pelo método do ácido acético/ nítrico, que consistiu na preparação de uma solução na proporção de 5 partes de ácido acético e 1 parte de ácido nítrico. A biomassa foi imersa nessa solução por um período de 6 horas a $100^{\circ} \mathrm{C}$, quando a individualização das fibras foi alcançada (BARRICHELO e FOEKEL, 1983). Após esse procedimento o material foi posto em suspensão e agitado lentamente com auxílio de agitador magnético. Com o uso de microscópio óptico acoplado e uma câmera de alta resolução, realizou-se a análise morfológica de 50 fibras por lâmina, utilizando-se 4 lâminas distintas.

Com as dimensões obtidas, calculou-se o índice de enfeltramento - IE (Equação 1), coeficiente de flexibilidade CF (Equação 2), fração parede - FP (Equação 3) e índice de Runkel - IR (Equação 4). Esses parâmetros permitem estimar o comportamento do papel posteriormente produzido a partir da polpa, que deve ser confirmado a partir de testes físicomecânicos do papel produzido. $\mathrm{O}$ índice de enfeltramento e fração parede são utilizados para análise da resistência ao rasgo. Já o coeficiente de flexibilidade e índice de Runkel relacionamse à resistência à tração e arrebentamento, sendo que o IR ainda permite dizer o quão bom o material fibroso é para a produção de papel, de forma geral.

$$
\begin{aligned}
& I E=\frac{C}{L x 100} \\
& C F=\frac{D L}{L} \times 100 \\
& F P=\frac{2 x E P}{L} \times 100 \\
& I R=\frac{2 x E P}{D L}
\end{aligned}
$$

Onde: EP é a espessura de parede das fibras, DL é o diâmetro do lúmen, $\mathrm{L}$ é a largura das fibras e $\mathrm{C}$ é o comprimento das fibras.

\subsection{Caracterização química}

A caracterização química de $B$. vulgaris foi realizada a partir de uma série de procedimentos normatizados já existentes e comumente usados em estudos de química de biomassas lignocelulósicas, apresentados na Tabela 1.

Tabela 1 - Metodologias para caracterização química das amostras de bambu

\begin{tabular}{cc}
\hline Parâmetro & Procedimento \\
\hline Classificação dos cavacos & SCAN 40:94 \\
Extrativos totais & TAPPI T 264 cm-97 \\
Lignina insolúvel & TAPPI T222 cm-11 \\
Lignina solúvel & Goldschimid (1971) \\
Hidrólise ácida & TAPPI T 249 cm-85 \\
Composição de carboidratos & WALLIS et al. $(1996)$ \\
Ácidos urônicos & SCOTT (1979) \\
Grupos acetil & SOLAR et al. (1987) \\
Cinzas & TAPPI T264 cm-97 \\
\hline
\end{tabular}

\subsection{Extração de amido}

A extração de amido deu-se pelo método de desfibramento mecânico dos cavacos seguido de extração por arraste em água, proposto por Azzini (1984). Os cavacos aceitos após classificação foram desintegrados em moinho de martelo, de forma que os grânulos de amido fossem extraídos das células parenquimáticas. Em seguida, o bambu desfibrado em moinho foi, então, batido por 15 segundos em liquidificador doméstico com água na proporção 1:10. O bambu desfibrado e livre de amido foi seco e estocado para preparação de polpa celulósica por polpação kraft.

\subsection{Polpação kraft do bambu}

As etapas de vaporização, impregnação e cozimento foram realizadas em digestor $\mathrm{MK}$, utilizando-se o material fibroso livre de amido. As condições gerais utilizadas estão apresentadas na Tabela 2. A performance da conversão do bambu em polpa celulósica foi avaliada pelo rendimento da polpação, determinado por gravimetria, e pela determinação do número kappa (TAPPI T236).

Tabela 2 - Condições gerais estabelecidas para a polpação kraft de bambu

\begin{tabular}{cc} 
Parâmetros & Resultados \\
Relação Licor / Biomassa & 4 \\
Álcali efetivo, \% NaOH & 9,8 \\
Sulfidez $(\%)$ & 35 \\
Temperatura máxima $\left({ }^{\circ} \mathrm{C}\right)$ & 170 \\
Tempo até temperatura máxima (min) & 90 \\
Tempo na temperatura máxima $(\mathrm{min})$ & 90 \\
\hline
\end{tabular}

\section{RESULTADOS E DISCUSSÃO}

\subsection{Morfologia do bambu}

Os resultados da morfologia de $B$. vulgaris encontram-se dispostos na Tabela 3. Obteve-se comprimento de fibra médio de 2,38 mm, comparável ao valor obtido por Guimarães Junior (2010) e inferior ao obtido por Gomide et al. (1981), que obtiveram 2,8mm. Esse valor também está de acordo com os estudos de Philip (1988), que afirma que os comprimentos predominantes se encontram entre 2,2 e 2,6 $\mathrm{mm}$. De acordo com a COPANT (1974), pode-se classificar as fibras do B. vulgaris como intermediárias, uma vez que apresentam comprimento médio entre as fibras de eucalipto $(1,0 \mathrm{~mm})$ e pinus $(3,5 \mathrm{~mm})$. Esse comprimento já é suficientemente favorável para conferir propriedades mecânicas elevadas aos produtos de sua aplicação. Obteve-se ainda largura de $26,73 \mu \mathrm{m}$, enquanto Gomide et al. (1981) obtiveram 17,2 $\mu \mathrm{m}$ e Guimarães Junior (2010) obteve $13,33 \mu \mathrm{m}$.

Tabela 3 - Dimensões das fibras de bambu e relações das propriedades morfológicas

\begin{tabular}{cc|} 
Dimensão & Resultado \\
\hline Comprimento de fibra / mm & 2,38 \\
Largura da fibra / $\mu \mathrm{m}$ & 26,73 \\
Diâmetro do lúmen / $\mu \mathrm{m}$ & 15,31 \\
Espessura de parede / $\mu \mathrm{m}$ & 5,95 \\
\hline
\end{tabular}

Ainda que o bambu utilizado tenha apresentado largura mais elevada que a obtida na literatura, esse valor é consideravelmente menor que a largura média de fibras de pinus $(46,75 \mu \mathrm{m})$, o que se dá devido ao diâmetro de lúmen do bambu, que é cerca de 2 vezes menor que a madeira de pinus. Ter fibras estreitas garante ao bambu maior resistência ao ar, facilita a 
formação da folha e favorece a resistência à tração, uma vez que fibras mais estreitas permitem melhor conexão entre fibras (LIMA, 2017).

As relações de propriedades morfológicas calculadas a partir das Equações 1-4 estão dispostas na Tabela 4.

Tabela 4 - Relações das propriedades morfológicas de $B$. vulgaris

\begin{tabular}{cc|} 
Propriedade & Resultado \\
\hline Índice de enfeltramento & 89,16 \\
Coeficiente de flexibilidade (\%) & 57,28 \\
Fração parede (\%) & 44,53 \\
Comprimento / espessura & 400,45 \\
Índice de Runkel & 0,78 \\
\hline
\end{tabular}

A relação comprimento/largura (índice de enfeltramento) obtida foi de 89,16 , bem superior a outras biomassas lignocelulósicas de propriedades físico-mecânicas inferiores, como por exemplo o coco (35) e a cana-de-açúcar (66) (GUIMARÃES, 1984), indicando que as fibras são bastante flexíveis. Essa tendência também é observada a partir do coeficiente de flexibilidade $(57,28 \%)$ obtido para o bambu, que também é superior ao usualmente obtido em madeiras de pinus e indica que as fibras estão parcialmente colapsadas, com boa união entre elas. Esse coeficiente influencia diretamente na resistência à tração do papel. Como a fração parede obtida $(44,53 \%)$ foi acima de $40 \%$, considera-se que haverá boa resistência ao rasgo. Já o índice de Runkel obtido $(0,78)$ permite classificar o bambu como bom para papel (STAMM, 1964).

Nota-se, portanto, que as fibras do bambu utilizado apresentam características morfológicas que permitem obter propriedades físico-mecânicas próximas à madeira de pinus e certamente superiores à madeira de eucalipto e outros materiais lignocelulósicos que apresentam fibras curtas.

\subsection{Caracterização química do bambu}

Os resultados obtidos na caracterização química de $B$. vulgaris encontram-se dispostos na Tabela 5. Obteve-se 23,2\% de lignina total e $63,4 \%$ de holocelulose, que corresponde ao somatório de celulose e hemiceluloses na biomassa. Os valores estão próximos dos encontrados por Gomide (1986), que obteve $64,94 \%$ de holocelulose e $17,90 \%$ de lignina. O alto teor de holocelulose é uma vantagem do bambu para a produção de polpa celulósica, e seu alto teor de pentosanas $(16,6 \%)$ facilita o refino da polpa e eleva as características de resistência (GOMIDE et al., 1981).

Em média, madeiras de coníferas geralmente apresentam $69 \%$ de holocelulose e $28 \%$ de lignina, enquanto que madeiras de folhosas apresentam $75 \%$ de holocelulose e $20 \%$ de lignina (SJÖSTRÖM,1993; IRANMAHBOOB et al., 2002). Observase que a Bambusa vulgaris apresenta teor de lignina menor que as madeiras de coníferas e folhosas, o que é esperado para gramíneas (THOMAS, 1977). Entretanto, deve-se levar em consideração que uma parcela dessa lignina presente no bambu é lignina p-hidroxifenila, mais presente nesse tipo de cultura que nas coníferas, que possuem guaiacila, e folhosas, que possuem lignina do tipo siringila/guaiacila (SARKANEN e HERGERT, 1971). A p-hidroxifenila é a lignina menos reativa em meio alcalino entre as três, uma vez que seu precursor (ácido pcoumarílico) apresenta dois carbonos aromáticos livres e, portanto, dois sítios passíveis de ligações com outras unidades de fenilpropano, como, por exemplo, ligações carbono-carbono que são fortes, o que eleva o grau de condensação da lignina e torna mais difícil sua deslignificação em processos de polpação kraft para a produção de polpa voltada à indústria papeleira (GUTIÉRREZ et al., 2006). Nota-se, portanto, que o baixo teor de ligninas no bambu colabora para uma deslignificação mais fácil, mas a característica da lignina presente nas gramíneas desfavorece o processo de polpação. Gomide et al. (1981) observam ainda que colmos mais jovens são preferenciais, uma vez que o teor de lignina se eleva com o tempo.

\begin{tabular}{ccccc} 
Tabela 5 - Caracterização química de B. vulgaris & \\
\hline Parâmetro & & A & B & Média \\
\hline Extrativos totais / \% & & 8,9 & 8,9 & 8,9 \\
Cinzas / \% & 0,67 & 0,63 & 0,65 \\
& Insolúvel & 22,7 & 21,5 & 22,1 \\
Lignina Klason / \% & Solúvel & 1,1 & 1,1 & 1,1 \\
& Total & 23,8 & 22,6 & 23,2 \\
Grupos acetil / \% & & 2,8 & 2,6 & 2,7 \\
Ácidos urônicos / \% & & 1,1 & 1,1 & 1,1 \\
& Arabinanas & 0,9 & 0,9 & 0,9 \\
& Galactanas & 0,5 & 0,5 & 0,5 \\
Carboidratos / \% & Glicanas & 45,7 & 46,7 & 46,2 \\
& Xilanas & 15,4 & 15,9 & 15,7 \\
& Mananas & 0,1 & 0,1 & 0,1 \\
\hline
\end{tabular}

Obteve-se $0,65 \%$ de teor de cinzas, valor inferior aos obtidos por Gomide et al. (1981) para colmos variando de 1 a 5 anos (teores entre 2,2 e 3,2\%) e próximo dos usualmente obtidos para coníferas e folhosas. Segundo Lybeer (2006), entre 1,5 a $6,4 \%$ desse valor geralmente correspondem à sílica, o que é desfavorável para aplicação industrial, uma vez que sua presença afeta as propriedades de polpação e dificulta o corte. Gomide et al. (1981) ressaltam que a sílica dissolvida no licor de polpação, ao entrar em contato com íons cálcio, deposita nas paredes dos tubos da caldeira de recuperação, ocasionando perda de eficiência na transferência de calor. O teor de cinzas também é composto principalmente por cálcio, potássio, manganês, magnésio, zinco, cobre, ferro e alumínio (TAMOLANG et al., 1980). Deve-se notar, entretanto, que teores de cinzas não são absolutos e variam com a idade dos colmos, havendo redução com o tempo, como observado por Chen et al. (1985) em estudo realizado com bambus de diferentes idades da espécie Phyllostachys pubescens. Obter um valor baixo de cinzas é, portanto, favorável para a produção kraft de polpa celulósica analisando-se apenas pelo ponto de vista da sílica. Todavia, como o teor de cinzas também é um indicativo de nutrientes, pode significar que o manejo do solo e a disponibilidade de elementos essenciais estão insuficientes, prejudicando o ciclo produtivo do bambu, o que influencia também na qualidade dos colmos colhidos. De forma geral, o teor de cinzas é usualmente mais elevado em gramíneas do que em coníferas e folhosas (GUIMARÃES JUNIOR et al., 2011), diferentemente do que foi observado nesse estudo.

Extrativos são desfavoráveis no processo polpação, pois estão associados com incrustações em equipamentos, além de reagirem com as soluções de cozimento consumindo álcali, e sendo os produtos dessas reações também adversos, como por exemplo, em reações de saponificação de ácidos graxos que 
formam espumas nos sistemas prejudicando etapas de processo como a lavagem da polpa. Os extrativos ainda se correlacionam com a formação de pitch na polpa, os quais são denominados como acúmulos de substâncias de origem lipofílica que se depositam sobre a polpa celulósica. Obteve-se, nesse estudo, média de $8,9 \%$ de extrativos totais, valor bem próximo ao obtido por Gomide et al. (1981) em bambus da mesma espécie com 5 anos $(9,0 \%)$ e bem mais elevados do que a média obtida em coníferas e folhosas, em torno de 3,0\% (SJÖSTRÖM, 1993; IRANMAHBOOB et al., 2002).

As variações na caracterização química de amostras de uma mesma espécie colhidas em diferentes locais é comum e justifica valores às vezes divergentes dos obtidos em literatura (LWIN et al., 2000). A disponibilidade de nutrientes do solo em que a cultura é plantada, os fatores climáticos, como radiação solar e índice pluviométrico e até mesmo a idade em que os colmos são colhidos são alguns dos fatores que afetam a composição química e justificam tais variações (QISHENG et al., 2001).

\subsection{Polpação kraft para produção de papel sackraft}

A polpação kraft do bambu livre de amido para obtenção de polpa celulósica visando a produção de papel sackraft (kappa $67,8)$ obteve rendimento total de $54,9 \%$. O rendimento é competitivo quando comparado a dados da literatura. Resultados obtidos, por exemplo, por Andrades e Frinhani (2015) para obtenção de polpa kappa 55 para Pinus taeda indicam rendimento da ordem de $52,5 \%$. Outro estudo realizado para Pinus taeda em kappa 65,9 apresentou rendimento da ordem de 50,09\% (COELHO, 2017).

Gomide et al. (1982) enfatizam, entretanto, que o rendimento de polpação do bambu é usualmente menor que de espécies coníferas em virtude do alto teor de sílica e de extrativos, ressaltando ainda que isso pode ser compensado devido a sua alta produtividade por unidade de área em comparação às madeiras convencionais. $\mathrm{O}$ bom rendimento desse estudo pode ser atribuído, portanto, ao baixo teor de sílica presente nos colmos.

A remoção do amido anteriormente à polpação também auxiliou para que o rendimento total fosse tão elevado. Gomide et al. (1988) comprova essa relação ao realizar a conversão de B. vulgaris sem extração do amido em polpa celulósica com rendimento de $365 \mathrm{~g} \mathrm{~kg}^{-1}$, enquanto que Pinus elliotti acarretou em $501 \mathrm{~g} \mathrm{~kg}^{-1}$ de celulose.

\section{CONCLUSÕES}

A partir das análises realizadas e dos resultados observados, conclui-se que o bambu é uma biomassa que pode substituir a madeira na aplicação sugerida. As fibras de bambu possuem morfologia semelhante à do pinus e os teores de holocelulose e lignina também são parecidos com a da madeira, no que se conclui que o bambu da espécie B. vulgaris possui potencial para a obtenção de papeis de embalagem com propriedades físico-mecânicas semelhantes às obtidas com coníferas.

É possível extrair amido do bambu a partir da metodologia de desfibramento mecânico de cavacos seguido por arraste em água, acarretando na otimização do rendimento da polpação.

Foi possível produzir polpa celulósica kraft com kappa elevado, voltada à utilização para fabricação de papeis de embalagem sackraft. Os rendimentos obtidos são competitivos com os obtidos a partir da polpação realizada com coníferas, no que se conclui que o bambu pode, de fato, ser utilizado com sucesso.

\section{AGRADECIMENTOS}

Os autores agradecem o suporte financeiro concedido pelo Conselho Nacional de Desenvolvimento Científico e Tecnológico (CNPq).

\section{R E F E R ÊN C I A S}

ANDRADES, C.; FRINHANI, E.M. Propriedades físicomecânicas de polpas kraft com números kappa 45 e 55 destinadas a fabricação de papel sackraft de baixa gramatura. O PAPEL, v. 76, n. 7, p. 53-58, 2015.

AZZINI, A. Amido a partir de bambu. Bragantia, v.43, n.1, p.4550, 1984.

AZZINI, A.; ARRUDA, M. C. Q. de; CIARAMELLO, D.; SALGADO, A. L. de B. e TOMAZELLO FILHO, M. Produção conjunta de fibras celulósicas e etanol a partir do bambu. Bragantia, v.46, n.1, p.17-25, 1987.

BARRICHELO, L.E.G.; FOELKEL, C.E.B. Processo nítricoacético para maceração de madeira. In: CONGRESSO FLORESTAL BRASILEIRO, 4.ed, 1982, Belo Horizonte. Anais. São Paulo: Sociedade Brasileira de Silvicultura, 1983. p.732-733.

BERALDO, A. L.; AZZINI, A. Bambu: características e aplicações. Editora Guaíba: Agropecuária, 2004. Guaíba - RS, 2004.128p.

BRÄNNVALL, E; GÖRAN, A. Pulp Characterisation. In: Pulping Chemistry and Technology. 1ed: Berlin, Walter de Gruyter, v.2, p. 429-460, 2009.

CHEN, Y. D.; QIN, W. L. The chemical composition of ten bamboo species. In: Proceedings of the International Bamboo Workshop, Hangzhou, China, 6-14 October 1985. Anais... Chinese Academy of Forestry, Beijing, China; International Development Research Centre, Ottawa, Canada. p.110-113, 1985.

COELHO, M. U. Avaliação tecnológica da madeira de Pinus maximinoi visando sua utilização industrial na produção de celulose kraft. 2017. 94f. Dissertação (Mestrado em Ciências) - Programa de Pós-Graduação em Recursos Florestais, Universidade de São Paulo, Piracicaba, 2017.

COPANT, 1974. Comisión Panamericana de Normas Técnicas - COPANT. Descripción de características generales, macroscópicas de las maderas angiospermas dicotiledóneas. COPANT 1974; 30: 1-19.

CUNHA, S. D.; GRUPIONI, P. H. F.; SILVA, A. C. S.; ARAÚJO, M. S.; CONEGLIAN, A. Viabilidade econômica do plantio de eucalipto VM01 para produção de lenha. Enciclopédia Biosfera, v. 15, n. 27, p. 145-154, 2018.

FILGUEIRAS, T.S. e GONÇALVES, A.P.S. A checklist of the Basal Grasses and Bamboos in Brazil (Poaceae). Bamboo Science \& Culture. The Journal of the American Bamboo Society. Vol. 18.2004. p7-18

GOLDSCHMID, O. Ultraviolet spectra, In: Lignins: 
occurrence, formation, structure and reactions, Sarkanen, K. V. and Ludwig, C. H. (eds), Wiley-Interscience, New York, 241-266, 1971.

GOMIDE, J. L. Estudo sobre a constituição química do Bambusa vulgaris, visando a produção de polpa celulósica. O papel, v.47, n.10, p.64-68, 1986.

GOMIDE, J. L.; OLIVEIRA, R. C; COLODETTE, J. L. Influencia da idade do Bambusa vulgaris nas suas características químicas a anatômicas visando a produção de polpa celulósica. In: Congresso Anual da ABCP, 14, 1981, São Paulo, v. 1, p. 5-29.

GOMIDE, J. L.; COLODETTE, J. L.; OlIVEIRA, R. C. Caracterização química e anatômica do Bambusa vulgaris visando à produção de polpa celulósica. Viçosa, SIF, (1981), 22 pag. (Relatório Técnico).

GOMIDE, J. L.; COLODETTE, J. L.; OlIVEIRA, R. C. Influência do álcali ativo e da temperatura na polpaçao kraft de Bambusa vulgaris. In: Congresso Anual da ABCP, 15, 1982, São Paulo, v. 1, p. 189-203.

GOMIDE, J. L.; VIVONE, R. R.; GALA, P. A. M. Bambu: uma alterativa para o déficit de celulose de fibra longa no Brasil. In: Congresso Anual da ABCP, 21, 1988, São Paulo, v. 1, p. 1-16.

GUIMARÃES JUNIOR, M.; NOVACK, K. M.; BOTARO, V. R. Caracterização anatômica da fibra de bambu (Bambusa vulgaris) visando sua utilização em compósitos poliméricos. Revista Iberoamericana de Polímeros, v. 11, p. 442-456, dezembro de 2010.

GUIMARÃES JUNIOR, M.; NOVACK, K. M.; BOTARO, V. R.; PROTÁSIO, T. de P.; COUTO, A. M. Caracterização físico-química de fibra e polpas de Bambusa vulgaris Schrad para utilização em compósitos poliméricos. Revista Latinoamericana de Metalurgia y Materiales, v. 33, p. 33-42, novembro de 2011.

GUIMARÃES, S. Experimental mixing and moulding with vegetable fibre reinforced cement composites. International Conference on Development of Low-Cost and Energy Saving Construction Materials, Lehigh Valley, Pennsylvania, pp. 37-42, (1984).

GUTIÉRREZ, A.; RODRÍGUEZ, I. M.; DEL RIO, J.C. Chemical characterization of lignin and lipid fractions in industrial hemp bast fibers used for manufacturing highquality paper. Journal of Agriculture and Food Chemistry, v.54, p.2138-2144, 2006.

IBÁ, 2017. Relatório Anual 2017 / Indústria Brasileira de Árvores (IBÁ). - Brasília: 2017. 80 p. Disponível em <http://iba.org/images/shared/Biblioteca/IBA_Relatorio Anual2017.pdf $>$. Acesso em 20 de junho de 2018.

ILVESSALO-PFAFFLI, M. -S. Fiber Atlas - Identification of papermaking fibers. Springer: Heidelberg. 1995. 316p.

IRANMAHBOOB, J.; NADIM, F.; MONEMI, S. Optimizing acid-hydrolysis: a critical step for production of ethanol from mixed wood chips. Biomass and Bioenergy, v. 22, p. 401-404, 2002

JUDZIEWICZ, E.J. et al. American bamboos. Washington, D.C.: Smithsonian Institution Press. 1999. $392 \mathrm{p}$

LIMA, D. O.; GOMES, F. J. B. ; LATORRACA, J. V. F. ; SANTOS, G. C. V. ; PACE, J. H. C. ; RAMOS, L. M. A. ; CASTOR NETO, T. C. ; CAHUANA, L. A. P. . Evaluation of ten eucalypt species regarding their fiber morphology aiming paper quality. In: 9th International Symposium on Wood, Fiber and Pulping Chemistry, 2017, Porto Seguro. 19th International Symposium on
Wood, Fiber and Pulping Chemistry.

LWIN, K. M.; HAN, Y.Y.; MAUNG, K. W.; MOE, A. Z.; THAN, S. B. M. An investigation on morphology, anatomy and chemical properties of some Myanmar bamboos. In: Proceedings of Myanmar Academy of Agriculture and Forestry, p.1-18, 2000.

LYBEER, B. Age-related anatomical aspects of some temperate and tropical bamboo culms (Poaceae: Bambusoideae). 2006. V.1, 201p. Thesis (PhD in Biology) - Ghent University, Belgium, 2006.

OLIVEIRA, L. F. A. Conhecendo Bambus e suas Potencialidades para uso na Construção Civil. 2013. 90f. Monografia (Curso de Especialização em Engenharia Civil) Escola de Engenharia, Universidade Federal de Minas Gerais, Belo Horizonte, 2013.

ORTHEY, A. L. Uso do bambu industrializado no Brasil e sua aplicação no design de móveis: Estudo de caso da empresa Oré Brasil. 2015. 130f. Dissertação (Mestrado em Design) - Programa de Pós-Graduação em Engenharia Civil, Universidade Federal de Viçosa, Viçosa, 2017.

OSTAPIV, F. Análise e melhoria do processo produtivo de tábuas de bambu (Phyllostachys pubescens) com foco em pisos. 2007. 112f. Dissertação (Mestrado em Engenharia) - Setor de Artes, Comunicação e Design, Universidade Federal do Paraná, Curitiba, 2015. PEREIRA, M. A. R. Projeto Bambu: Introdução de Espécies, Manejo, Características e Aplicações. 2012. 210f. Tese (Título de Livre-Docente em Design e Construção com Bambu) - Faculdade de Engenharia de Bauru, Universidade Estadual Paulista "Julio de Mesquita Filho, Bauru, 2012.

PEREIRA, M. A. dos R.; BERALDO, A. L. Bambu de corpo e alma. Bauru, SP: Canal6, 240 p., 2007.

PHILLIP, P. Celulose e papel; vol. I: Tecnologia da fabricação de pasta celulósica; vol.II: Tecnologia da fabricação do papel, $2^{\text {a }}$ ed., IPT, 1988.

QISHENG, Z.; SHENXUE, J.; YONGYU T. Industrial utilization on bamboo. International Network for Bamboo and Rattan (INBAR). Technical Report, n.26. Beijing, Republic of China, 2001.

REUBENS, R. Bamboo in Sustainable Contemporary Design. INBAR Working Paper, Beijing, n.60, 2010.

SARKANEN, K.V; HERGERT, H.L. Lignins: occurrence, formation, struture and reaction. New York: Wiley, 1971.

SCANDINAVIAN PULP, PAPER AND BOARD TESTING COMMITTEE. Scan Test Methods, 1993.

SCOTT, R.W., Colorimetric determination of hexuronic acids in plant materials. Analytical Chemistry. 51(7):936941,1979 .

SILVA, C. A.F.; BUENO, J. M.; NEVES, M. R. A Indústria de Celulose e Papel no Brasil. Guia ABTCP: Fornecedores \& Fabricantes. Disponível em: < http://www.poyry.com.br/sites/www.poyry.com.br/files/ media/related_material/16out27a-abtcp.pdf > acesso em 21 de junho de 2018.

SJÖSTRÖM, E. Wood chemistry: fundamentals and application. London: Academic Press, 1993. 293 p.

SOLAR, R., F. KACIK,, I. MELCER. Simple semi-micro method for the determination of o-acetyl groups in wood and related materials. Nordic Pulp and Paper Research Journal, 4:139-141, 1987. 
STAMM, A. J. Wood and cellulose science. The Ronald Press Company. New York, 1964. 549p.

TAMOLANG, F. N.; LOPEZ, F. R.; SEMANA, J. A.; CASIN, R. F.; ESPILOY, Z. B. Properties and utilization of Philippine erect bamboos. Bamboo research in Asia. In: Proceedings of a Workshop held in Singapore, 1980, Ottawa. Anais... 1980. p.189-200.

TECHNICAL ASSOCIATION OF THE PULP AND PAPER INDUSTRY-TAPPI. Tappi test methods: 1992-1993. Atlanta, 1992.

THOMAS, R. J., Wood structure and chemical composition. In: GOLDSTEIN, I. S. Wood technology: chemical aspects. Washington, D. C.: American Chemical Society, 1977. p.1-23. (ACS Symposium Series, 43).

WALLIS, A.F.A., WEARNE, R.H., WRIGHT, P.J., Chemical analysis of polysaccharides in plantation eucalypt woods and pulps. Appita Journal 49(4):258-262, 1996.

XUHE, C. Promotion of Bamboo for Poverty Alleviation and Economic Development. In: Proceeding of International Workshop on Bamboo Industrial Utilizations, October, 2003: International Network for bamboo and RattanINBAR, Nanjing Forestry University of China, 2003.

ZEHUI, J. Characteristic and Prospects for Processing Approaches for Bamboo Resources IN: PROCEEDING OF INTERNATIONAL WORKSHOP ON BAMBOO INDUSTRIAL UTILIZATIONS. International Network for bamboo and Rattan-INBAR, October, 2003. 